\title{
Beating classical imaging limits with entangled photons
}

\section{Miles Padgett, Ermes Toninelli, Thomas Gregory, Paul- Antoine Moreau}

Miles Padgett, Ermes Toninelli, Thomas Gregory, Paul-Antoine Moreau, "Beating classical imaging limits with entangled photons," Proc. SPIE 10934, Optical, Opto-Atomic, and Entanglement-Enhanced Precision Metrology, 109341R (1 March 2019); doi: 10.1117/12.2515497

SPIE. Event: SPIE OPTO, 2019, San Francisco, California, United States 


\title{
Beating classical imaging limits with entangled photons
}

\author{
Miles Padgett, Ermes Toninelli, Thomas Gregory and Paul-Antoine Moreau \\ School of Physics and Astronomy, University of Glasgow, Glasgow. G12 8QQ, UK.
}

\begin{abstract}
How can quantum mechanics deliver better imaging performance? Parametric down-conversion sources produce pairs of photons that are correlated in many degrees of freedom, including their spatial positions. By using a camera to detect these pairs of photons it is possible configure imaging systems that can either beat the classical resolution limit or the classical noise limit. We demonstrate how a simple down-conversion source based on a laser and non-linear crystal can be combined with an EMCCD camera to achieve either of these outcomes. Firstly, when both photons pass through the sample, we show a full-field, resolution-enhancing scheme, based on the centroid estimation of the photon pairs. By optimising the software control of the EMCCD camera running in the photon-sparse regime we achieve a resolution enhancement over the equivalent classical limit. Secondly, we show a similar scheme but where only one of the two photons pass through the sample and the other acts as a reference, in this case the ratio of the two resulting images eliminates the background noise of the camera, and background light, achieving an increase in image contrast.
\end{abstract}

Keywords: Imaging, Quantum Correlations, Entanglement.

\section{INTRODUCTION}

In the 1990's Shih ${ }^{1}$ and others pioneered the use of parametric down-conversion for the production of photons pairs, using them as the illumination source for imaging systems. Those early systems typically used one of the photons to illuminate the object and a single-element detector was used to detect those photons which were transmitted, whilst the other photon, which had never interacted with the object, was directed to an imaging detector. The single-element detector reveals no spatial information whereas the imaging detector reveals no information about the object. However, because the two separated photons are correlated in their lateral position then a correlation between the single-element and the imaging detectors does reveal an image of the object. This separation of the object from the camera leads such systems to be referred to as "ghost imaging" systems. For a number of years a debate ensued as to whether this form of imaging was dependent upon quantum correlations or whether similar results could be obtained using classical light sources $^{2}$. Largely, the consensus view is now that similar imaging results can be obtained using spatial correlations that are either classical or quantum in nature ${ }^{3,4}$.

Although the separation of the object from the imaging detector itself is intriguing we report here two alternative configurations where both of the photons are detected by the same imaging detector. By using a camera to detect both photons it is possible configure imaging systems that can either beat the classical resolution limit or the classical noise limit. We demonstrate how a simple down-conversion source based on a laser and non-linear crystal can be combined with an EMCCD camera and appropriate software to achieve either of these outcomes.

\section{DETECTION OF THE PHOTON PAIRS WITH EMCCD CAMERA}

Many of the imaging systems based on down-conversion sources that have been demonstrated to date have suffered from the same practical flaw, namely that the imaging detector is a raster-scanned, single-element detector which reduces the overall effective quantum efficiency of the system in proportion to the number of pixels in the image ${ }^{5}$. Ideally one would wish to use a detector array as the imaging detector; however, until recently the cumulative noise of such arrays was too high to measure reliably the position of a single photon within the field of view. A number of groups have now shown that it is possible to use EMCCD-based cameras to detect both photons in the pair and distinguish these from both stray background photons and detector noise events ${ }^{6,7}$. The EMCCD is typified by having a very high quantum detection efficiency $(>80 \%)$ but suffers from a read-out noise resulting from the clock-induced charge $(\approx 0.005 /$ pixel $)$. In our own previous work we have used an EMCCD to detect either the strong position-correlation of photon-pairs in the image plane of the down-conversion crystal or the strong position anti-correlation of photon pairs in the far-field of the crystal $^{6}$. 
In response to the absorption of a photon within the active area of a single pixel, the EMCCD amplifies the number of corresponding electrons from one to many as part of the read-out process as the charge is passed from pixel to pixel. The result is that after read-out every pixel in the image has an analogue voltage associated with it, the more photons absorbed in the original pixel, the higher the voltage associated. However, the consistency by which the voltage can be related to the number of photons is not high, making photon number resolved estimates difficult ${ }^{8}$. Despite this difficulty, if the signal and idler outputs from a parametric down-conversion source are directed to different regions of the sensor, it is possible to observe multi-photon ( $>1 /$ pixel) sub-shot noise spatial correlation within the detected signals 9. Such correlations have formed the bases of sub shot-noise imaging systems where the object is placed in one of the two down-converted beams whist the other acts as the photon-number correlated reference ${ }^{10}$. In our present work we are interested in measurements at the single photon/pixel level and hence set a threshold on the analogue output of the EMCCD, above which we assign it as a photon detection event. In most of our own experiments we aim to operate at a light flux where the number of real photons detected is comparable to the background noise event rate of the sensor $(\approx 0.005 /$ pixel $)$. We find this is the flux regime where the strength of the resulting correlations between the pairs of photons is strongest ${ }^{11}$.

\section{EXPERIMENTAL CONFIGURATION.}

In our present work we use a quasi-CW Nd:YAG-based laser, which is internally frequency-tripled to give upto $100 \mathrm{~mW}$ of light at $\lambda_{p}=355 \mathrm{~nm}$. This output beam is collimated to $1 \mathrm{~mm}$ in diameter and used to pump an $L_{z}=3 \mathrm{~mm}$ thick BBO crystal in either type-I or type-II phase-matching configuration to give degenerate signal and idler outputs at $710 \mathrm{~nm}$. The residual transmitted pump light is then blocked using a bandpass filter that passes only the down-converted photons. These signal and idler photons are strongly position correlated in the image plane of the crystal and strongly momentum anti-correlated, giving an anti-correlation in their positions in the far-field. This means that if an object is placed in an image plane of the object then both photons interact with the object but if the object is place in the far-field of the crystal where the photons are laterally separated from each other it is possible for the object to interact with one of the photons and not the other.

We are exploring two different configurations for using these photon pairs as the illumination source within an imaging system. The first of these (see figure 1) uses a type-I phase-matched crystal where the signal and idler photons are very strongly position correlation described by a correlation length, $\sigma_{x}$, given by $\sigma_{x} \approx \sqrt{L_{z} \lambda_{p} / 6 \pi}$. The plane of the crystal is imaged so that both photons interact with the object and are then imaged again onto the EMCCD sensor. However, the overall magnification is set such that the image is over resolved with the pixel size being somewhat smaller than the correlation length. Consequently, rather than both photons being detected within the same pixel it is more likely that the two photons are detected in near-by pixels such that it is possible to determine not just their individual positions but also the bisector of the positions. This identification of the bisector is only possible when the overall event rate is small such that the number of photons detected per pixel $<<1$ and hence an image requires the summation of the bisectors over many frames. The data, collected over many frames, can be assembled in two different ways to create an image of the object. The first of these images is simply the sum of all the detected events and has a resolution set by the diffraction limit of the imaging optics between the object and the EMCCD sensor. The second of these images is created from the sum of all the bisectors and should have a resolution which is improved by a factor of SQRT $2^{12}$. 


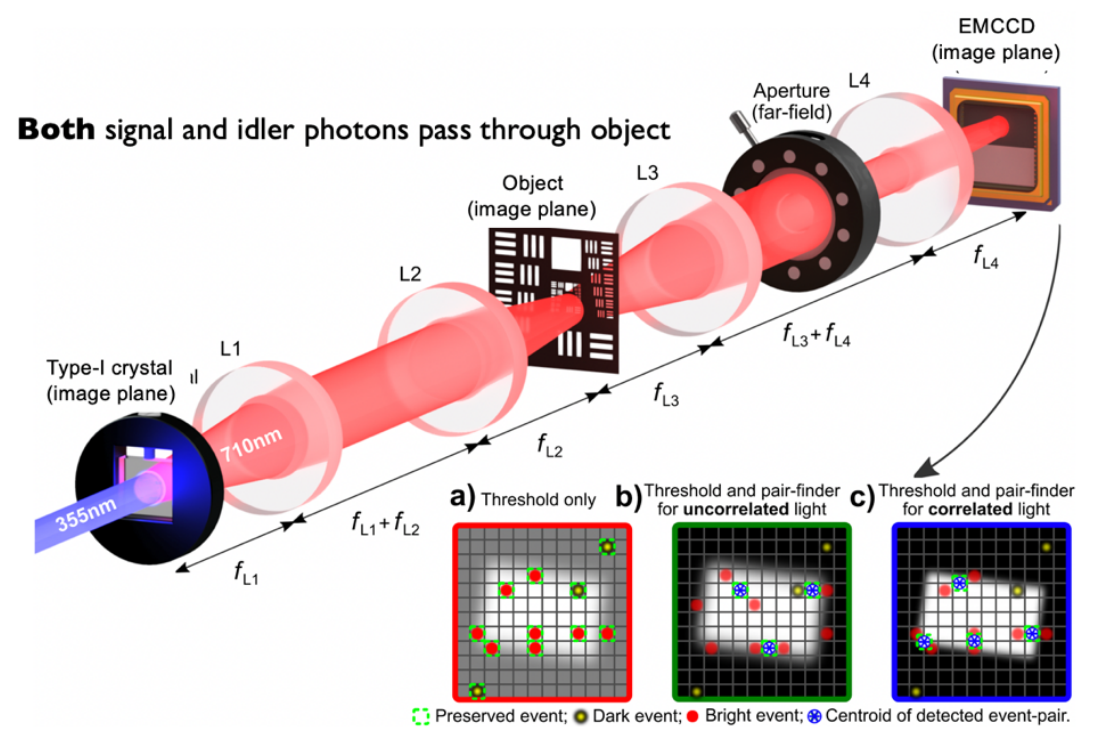

Figure 1. Experimental configuration for imaging based upon the bisectors of the signal and idler pairs.

The second configuration (see figure 2) for using these photon pairs as the illumination source within an imaging system uses a type-II phase-matched crystal. The birefringent nature of the non-linear crystal means that the signal and idler photon have an angular separation meaning that they are laterally shifted from each other in the far-field. In this far-field plane, the correlation length is given by $\sigma_{x} \approx f \lambda_{p} / 2 \pi w_{p}$, where $f$ is the effective focal length of the far-field lens and $w_{p}$ is the beam waist of the pump. The separation between the signal and idler photons means that one of them can illuminates the object whilst the other acts as a reference. The anti-correlation in the position of the signal and idler photons means that in order to compare the measured distribution of the photons, one of the distributions is laterally inverted, transforming the anti-correlation into a correlation. In order to maximise the strength of the correlations the overall magnification of the system is set such that the pixel size is somewhat larger than the correlation length. The resulting image of the object can then be formed by summing over many events. The conditional detection of the two photons suppresses both sensor noise and stray light from the final image. In essence this approach is the demonstration of the quantum illumination protocol proposed by Lloyd ${ }^{13}$. In principle, the degree to which the background light can be suppressed is unlimited, but is of course set by the technical restricitons of the detector, specifically its noise level since the noise events themselves can be accidently correlated with each other or with one of the baclground photons.

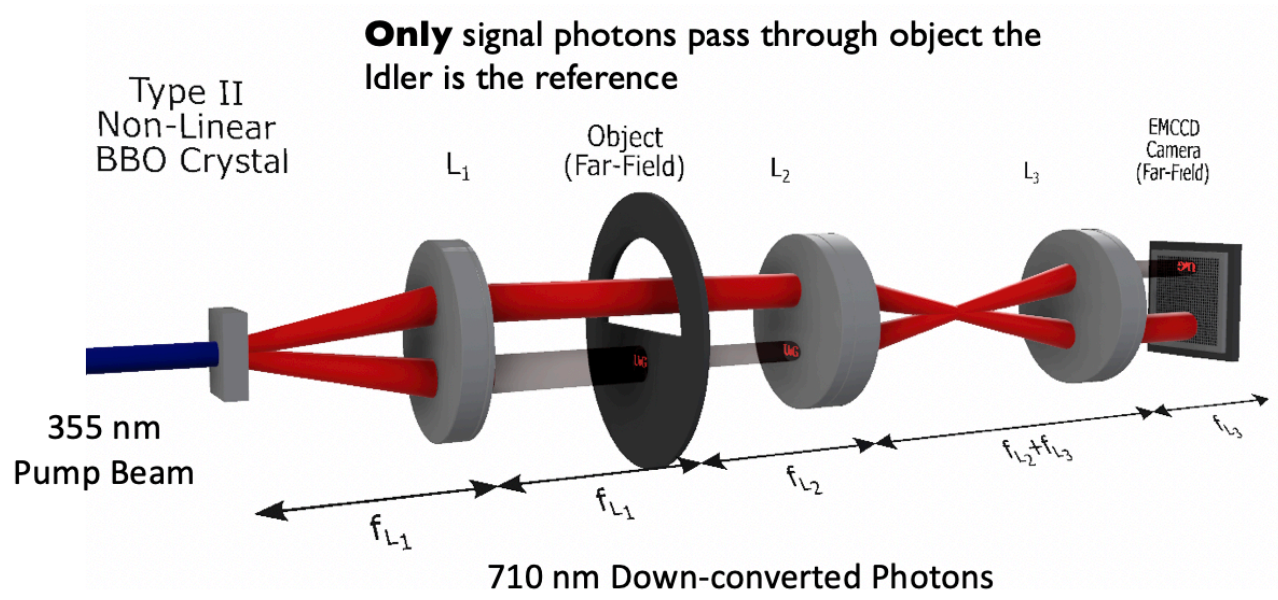

Figure 2. Experimental configuration for imaging based upon the quantum illumination protocol. 


\section{EXPERIMENTAL RESULTS.}

For the imaging based on bisectors, a critical aspect is to process each output frame of the EMCCD to give the maximal number of photon pairs whilst minimizing the number of events arising from the clock-induced-charge associated with the readout itself. However, it is also essential that the overall data is sparse enough to ensure that each pair event can be unambiguously identified through its spatial separation from near-by pair events. As discussed, we find that the optimum operating conditions correspond to a flux level where the number of photon-pair events is similar to the background noise of the sensor. Over an image size of several hundred pixels this typically yields $\approx 5$ bisector events per frame, therefore requiring $\approx 1,000,000$ frames, or more, to be summed together to acquire enough events to obtain a high-quality image. Figure 3 shows comparison images of a collection of paper fibres, one image is obtained from the sum of all sensor events (photons and noise are indistinguishable) and the other is the sum of only the bisector events. By visual inspection we see that the resolution of the bisector event image appears improved over the all events image. A detailed analysis reveals that in this current configuration we are obtaining only about half of the SQRT 2 predicted improvement, which modelling suggests is limited by the noise in our sensor leading to mis identification of photon pairs and hence the introduction of false bisector events. We are presently considering the use of other detector types, e.g. SPAD arrays which might aid the identification of the photons pairs through their temporal correlation and hence allow us to operate at a significantly higher event-rate.

For the imaging based on quantum illumination, a critical aspect is to ensure that the single (image) and idler (reference) photons are imaged to different regions of the EMCCD sensor but without any relative image distortion so that the spatial correlation is maintained over the full field-of-view. The detection of the image photons conditional on the reference is implemented through a simple AND-operation on the two image regions (after one of the image regions is laterally inverted), and summing these correlated events over many frames gives an image within which both the sensor noise and any background light is suppressed. Figure 3 shows comparison images of various objects, the top row being obtained from the quantum illumination protocol and the bottom from a simple sum of all events. By visual inspection we see the enhanced contrast arising from the quantum illumination. We are presently considering how such a scheme maybe modified to operate in situations where the level of background light is significant which, in order to keep the individual frames sparse, requires high frame-rates or short integration times suggesting again the use of SPAD arrays or, more conventionally, gated intensified detectors.

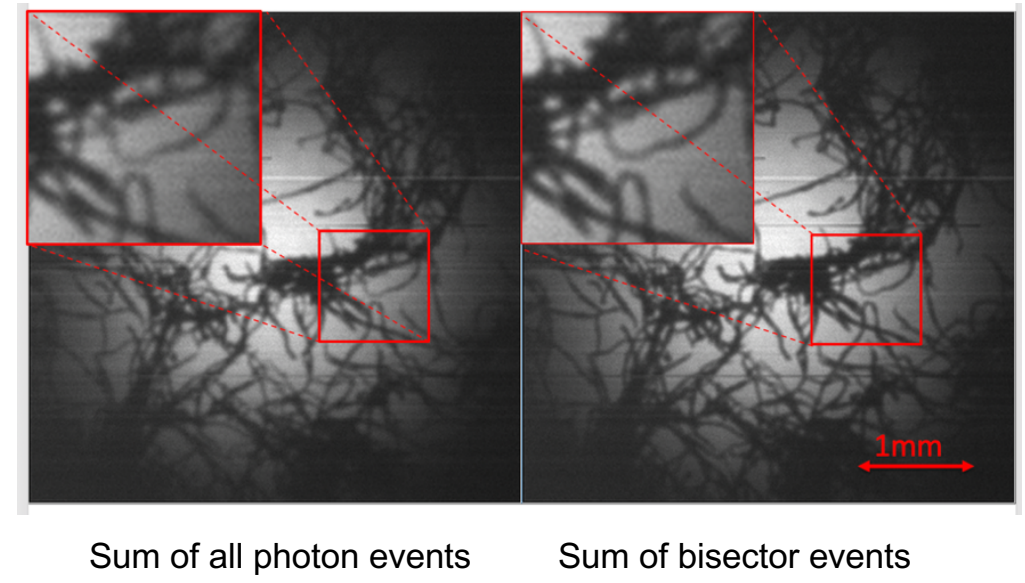

Figure 3. Images of paper fibres obtained by summing over all the detected photon events (left) and by summing all the bisectors of the photon pairs (right).

\section{SUMMARY.}

In this work we are seeking to explore how a comparatively simple experimental configuration based on commercial components, including off-the-shelf EMCCD detectors might be applied to the realizing the advantages of using photon pairs as the illumination source. By probing the object with both of photons it is possible to show an enhanced lateral resolution. By probing the object with one photon and making this detection conditional of the detection of the correlated photon it is possible to suppress both the background light and the sensor noise. This last configuration is an 
implementation of the Quantum Illumination Protocol ${ }^{13}$. Applications for these quantum imaging systems include the development of advanced microscopes, information security, and quantum LIDAR.

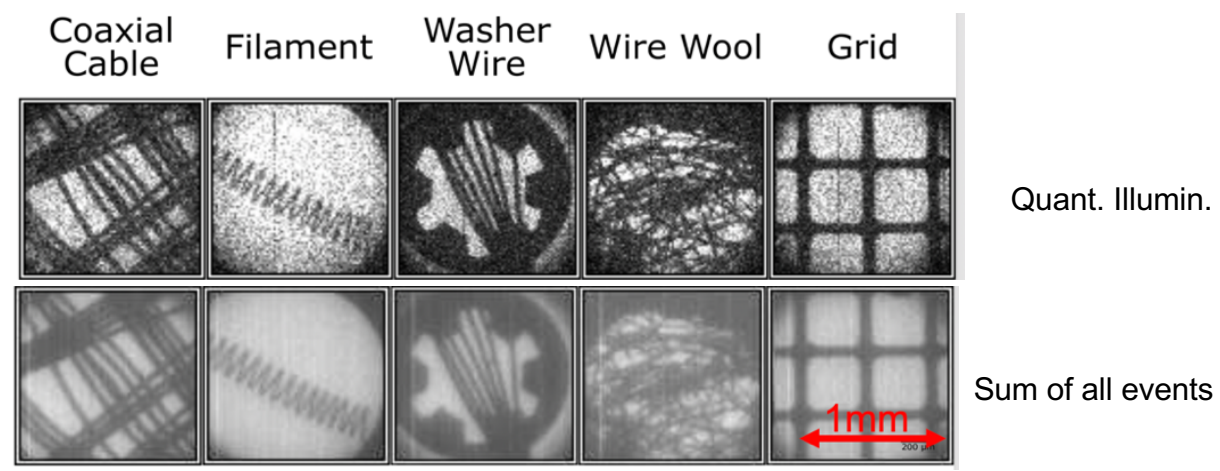

Figure 4. Images of various objects obtained through the quantum illumination protocol (top) and the sum of all detected events (bottom).

\section{REFERENCES}

[1] Pittman, T. B., Shih, Y. H., Strekalov, D. V., Sergienko, A. V., "Optical imaging by means of two-photon quantum entanglement," Phys Rev A 52(5), 3429-3432 (1995).

[2] Shapiro, J. H., Boyd, R. W., "The physics of ghost imaging," Quantum Inf Process 11(4), 949-993 (2012).

[3] Bennink, R. S., Bentley, S. J., Boyd, R. W., Howell, J. C., "Quantum and Classical Coincidence Imaging," Phys Rev Lett 92(3), 33601 (2004).

[4] Gatti, A., Brambilla, E., Bache, M., Lugiato, L. A., "Correlated imaging, quantum and classical," Phys Rev A 70(1), 013802 (2004).

[5] Aspden, R. S., Tasca, D. S., Boyd, R. W., Padgett, M. J., "EPR-based ghost imaging using a single-photon-sensitive camera," New J Phys 15(7), 073032 (2013).

[6] Edgar, M. P., Tasca, D. S., Izdebski, F., Warburton, R. E., Leach, J., Agnew, M., Buller, G. S., Boyd, R. W., Padgett, M. J., "Imaging high-dimensional spatial entanglement with a camera," Nat Commun 3, 984 (2012).

[7] Moreau, P.-A., Devaux, F., Lantz, E., "Einstein-Podolsky-Rosen Paradox in Twin Images," Phys Rev Lett 113(16), 160401-160405 (2014).

[8] Lantz, E., Blanchet, J.-L., Furfaro, L., Devaux, F., "Multi-imaging and Bayesian estimation for photon counting with EMCCDs," Monthly Notices RAS 386(4), 2262-2270 (2008).

[9] Jedrkiewicz, O., Jiang, Y. K., Brambilla, E., Gatti, A., Bache, M., Lugiato, L. A., Di Trapani, P., "Detection of sub-shot-noise spatial correlation in high-gain parametric down conversion," Phys Rev Lett 93(24), 243601 (2004).

[10] Brida, G., Genovese, M., Berchera, I. R., "Experimental realization of sub-shot-noise quantum imaging," Nat. Photonics 4, 227-230 (2010).

[11] Tasca, D. S., Edgar, M. P., Izdebski, F., Buller, G. S., Padgett, M. J., "Optimizing the use of detector arrays for measuring intensity correlations of photon pairs," Phys Rev A 88(1), 013816 (2013). 
[12] Tsang, M., "Quantum Imaging beyond the Diffraction Limit by Optical Centroid Measurements,” Phys Rev Lett 102(25), 253601 (2009).

[13] Lloyd, S., "Enhanced Sensitivity of Photodetection via Quantum Illumination," Science 321(5895), 1463-1465, American Association for the Advancement of Science (2008). 